\title{
Management of Post-Thyroidectomy Tracheomalacia
}

\author{
${ }^{1}$ Sabaretnam Mayilvaganan, ${ }^{2}$ Amit Agarwal
}

\begin{abstract}
Tracheomalacia is a heterogeneous and complex entity with no universally accepted definition and stratification. Historically, it is considered that patients with very large goiters are at risk of developing post-thyroidectomy tracheomalacia (PTTM). PTTM is a rare phenomenon and can be managed by either Noninvasive or invasive options.
\end{abstract}

Keywords: Tracheomalacia, Tracheostomy, Thyroid surgery.

How to cite this article: Mayilvaganan S, Agarwal A. Management of Post-Thyroidectomy Tracheomalacia. World J Endoc Surg 2014;6(2):96-98.

\section{Source of support: Nil}

Conflict of interest: None

\section{INTRODUCTION}

Tracheomalacia was first described by Czyhlarz in 1897, and remains a heterogeneous and complex entity with no universally accepted definition and stratification. Adult acquired tracheomalacia is most commonly post-traumatic due to prolonged or recurrent intubation. Historically, it is considered that patients with very large goiters were at risk of developing post-thyroidectomy tracheomalacia (PTTM). This condition is usually secondary to long-standing extrinsic tracheal compression with subsequent loss of tracheal cartilage rigidity, culminating in dynamic airway collapse in excess of $50 \%$ of diameter. It is commonly suggested that removal of the compressive source (i.e. thyroidectomy) may precipitate life-threatening airway collapse (particularly during expiration) and potentially mandate emergency tracheostomy. However, anecdotal evidence from surgeons with extensive experience suggests that this is almost never encountered and a rare phenomenon. ${ }^{1-5}$

\section{MANAGEMENT}

Tracheomalacia commonly affects the distal third of the trachea. Functional impairment is proportional to the length of the involved segment and degree of stenosis. Furthermore, kinking may occur at the transition between healthy tracheal wall and the indurated segment, as well as in the malacic

\footnotetext{
${ }^{1}$ Assistant Professor, ${ }^{2}$ Professor

1,2Department of Endocrine Surgery, Sanjay Gandhi Postgraduate Institute of Medical Sciences, Lucknow, Uttar Pradesh, India
}

Corresponding Author: Sabaretnam Mayilvaganan, Assistant Professor, Department of Endocrine Surgery, Sanjay Gandhi Postgraduate Institute of Medical Sciences, Lucknow, Uttar Pradesh India, Phone: 919655851510, e-mail: drretnam@gmail.com segment. In diffuse tracheal disease, the trachea usually distends and collapses unevenly during inspiration and expiration, thus interfering with the tracheal function (Fig. 1). ${ }^{1-3}$

Multiple preoperative risk factors in a patient with large goiter like goiter for more than 5 years (Fig. 2), preoperative recurrent laryngeal nerve palsy, significant tracheal narrowing and/or deviation, retrosternal extension, retro tracheal extension (Fig. 3), difficult tracheal intubation and thyroid cancer may be useful in predicting the need for planned tracheostomy following thyroidectomy. Although definitive criteria are lacking, a cut off of a $50 \%$ reduction in tracheal lumen is usually considered a prerequisite for diagnosis. This relies largely on dynamic imaging, either CT or MRI. However, in patients with extrinsic compression such as those due to goiter, tracheomalacia may theoretically only become apparent following removal of their compressive agent. Consequently, tracheomalacia may present unheralded as an emergency after thyroidectomy; hence historically, it has been feared as a complication of thyroidectomy. Though a number of investigations have been mentioned in the literature, in a postoperative patient a high clinical suspicion and bronchoscopy can be used for effective diagnosis and treatment. The airway is directly visualized during spontaneous respiration using a flexible bronchoscopy. The findings consist of the triad of loss of normal semicircular shape of tracheal lumen, forward ballooning of the posterior membranous wall and anteroposterior narrowing of the tracheal lumen. ${ }^{1-4,6,7}$

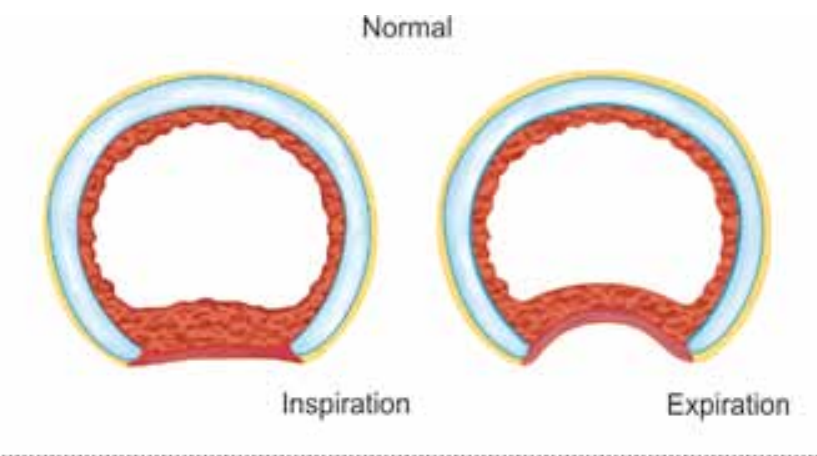

Tracheomalacia

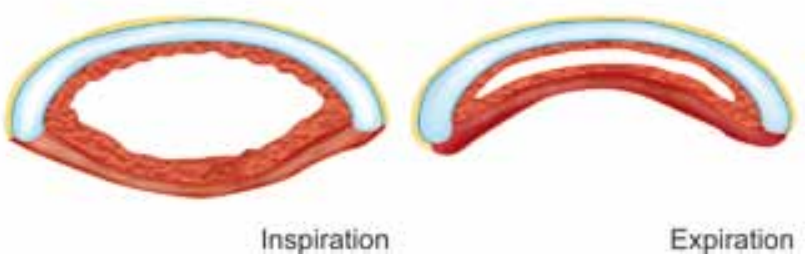

Fig. 1: Cross-section of normal trachea and tracheomalacia during inspiration and expiration 


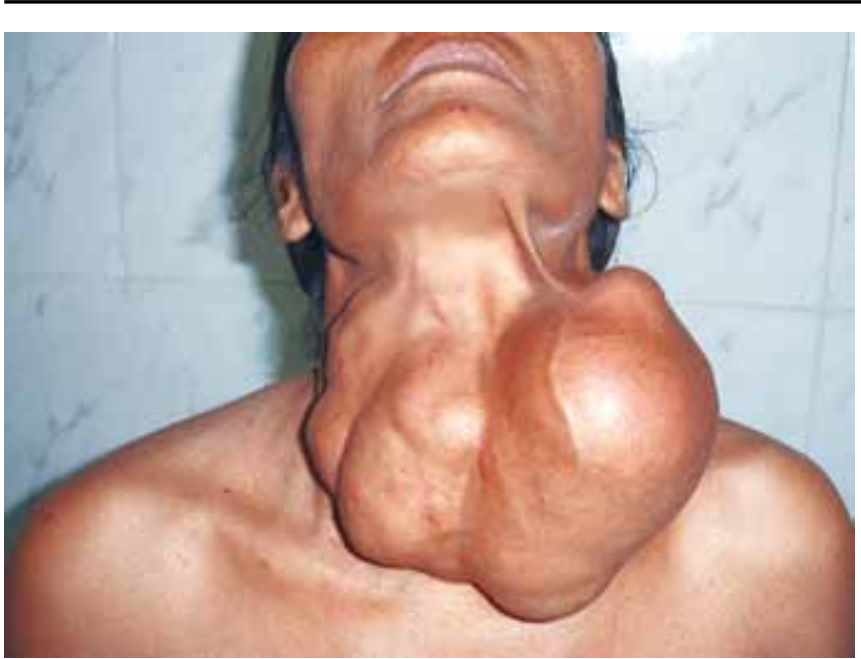

Fig. 2: Longstanding multinodular goiter

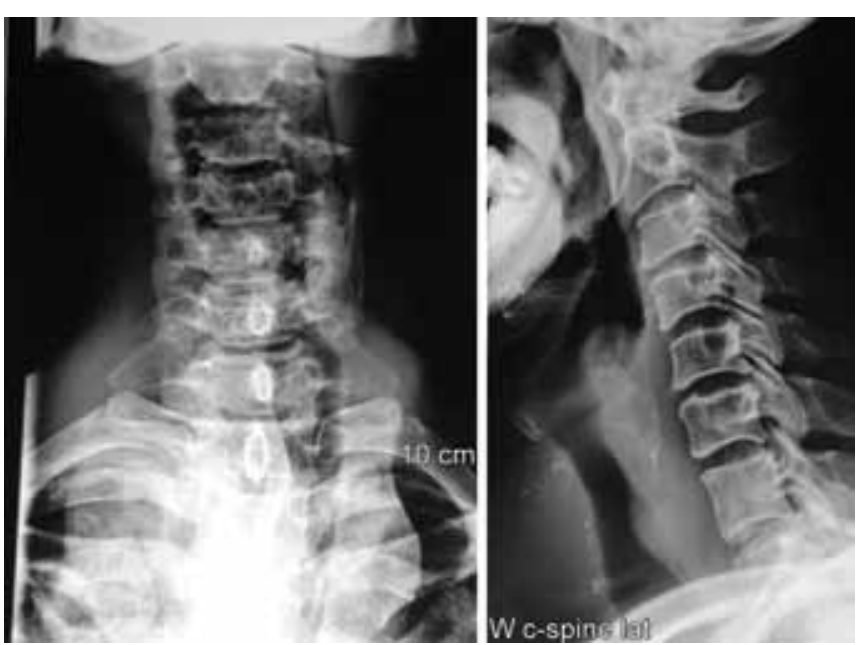

Fig. 3: X-ray showing tracheal narrowing and also retrotracheal extension

The important point is recognition of tracheomalacia on the operating table before extubation. There is no single foolproof criterion for confirming a diagnosis of tracheomalacia. However, for the intraoperative diagnosis of tracheomalacia, we have taken one or more of the following criteria:

- Soft and floppy trachea on palpation by the surgeon at the end of thyroidectomy. However, because of splinting effect of the endotracheal tube (ETT) in situ, it is difficult to appreciate a soft trachea. We therefore ask the anesthesiologist to gradually withdraw the tube for a short distance and then feel the tracheal without the tube in site. This maneuver may also help the surgeon recognize an obvious collapse of the tracheal wall.

- Obstruction to spontaneous respiration during gradual withdrawal of the ETT after thyroidectomy.

- Difficulty in negotiating the suction catheter beyond the ETT after gradual withdrawal.

- After closure of the wound, tracheomalacia can be suspected:

- If there is absence of peritubal leak on deflation of ETT cuff
- Volume pressure loop on ventilator or

- Development of respiratory stridor along with falling hemoglobin oxygen saturation $\left(\mathrm{SpO}_{2}\right)$ on pulse oximetry despite the administration of increasing $\mathrm{FiO}_{2}{ }^{5}$

The typical manifestation of postoperative tracheomalacia is acute respiratory distress following extubation not explained by any other cause. This invariably necessitates prompt re-intubation or a tracheostomy, following which the distress is promptly relieved. Anticipation of possible tracheomalacia helps in instituting preventive or prompt remedial measures. ${ }^{5}$

We recommend treating postoperative tracheomalacia following thyroidectomy expectantly. Mild variants, which may not be recognized postoperatively, may be managed with respiratory exercises. Mask ventilation using a CPAP mode may also be a useful adjunct. The majority will respond to conservative management, consisting of humidified air, chest physical therapy, slow and careful feedings to prevent aspiration, and control of infection and secretions with antibiotics.

\section{PROLONGED INTUBATION}

Patients who are unable to maintain oxygen saturation with these measures may be managed with intubation for longer periods. When extubation after about 2 weeks becomes a problem, we perform tracheostomy. The inflammatory response that sets in due to the indwelling tracheostomy or endotracheal tube leads to a stiffening of the tracheal wall thereby preventing the expiratory collapse. This is paradoxical since a long-standing tracheostomy tube can result in tracheomalacia because of pressure-related degeneration of the tracheal cartilages.

\section{TRACHEOSTOMY}

We prefer to go for an intraoperative tracheostomy if there is definite softening of the trachea ${ }^{6,7}$ we think that it is better to do a tracheostomy at the time of surgery, as it is easier to visualize the part of trachea most suitable for tracheostomy. Further, the tracheal toilet as well as ventilator care, if needed, is easier in patients with tracheostomy than in those with the ETT kept in place. Unlike prolonged intubation, tracheostomy results in fibrosis around a soft trachea resulting in early recovery from tracheomalacia. We also did not encounter any cases of tracheal stenosis following tracheostomy for tracheomalacia. Most of the tracheostomized patients had their tracheostomy tube removed after an week (Fig. 4A).

\section{TRACHEOPEXY}

We prefer this technique in short segment lateral tracheomalacia. We use fixation sutures to anchor the tracheal rings to 
both clavicles to maintain an anterolateral counter traction on the anterior wall of the trachea, so that it prevented its backward collapse and kept the airway patent ${ }^{8}$ (Fig. 4B).

\section{NONINVASIVE POSITIVE PRESSURE VENTILATION}

We prefer this technique in short segment tracheomalacia. The positive airway pressure delivered by NPPV can act as

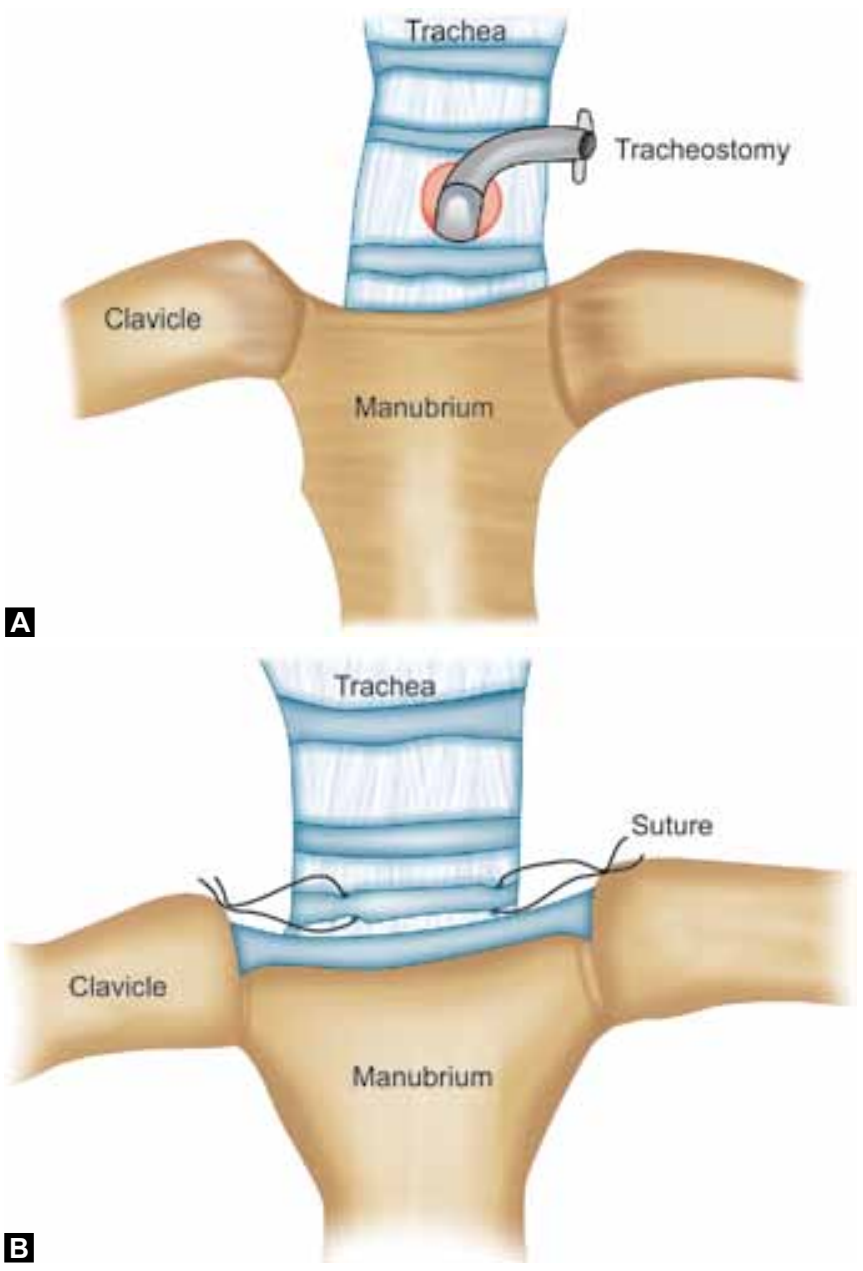

Figs 4A and B: (A) Tracheostomy and (B) Tracheopexy an internal stent and prevent tracheal collapse after withdrawal of the endotracheal tube in patients with tracheomalacia, thereby providing a potential therapeutic benefit. Noninvasive positive pressure ventilation with bi-level positive airway pressure mode delivers positive airway pressure both during inspiratory and expiratory phases. We use BIPAP ventilation for 48 hours. ${ }^{9}$

These are the techniques we usually use and we have little experience of using intraluminal tracheal splints and autologous costal cartilage ring grafts to support the trachea anteriorly.

\section{REFERENCES}

1. Lee C, Cooper RM, Goldstein D. Management of a patient with tracheomalacia and supraglottic obstruction after thyroid surgery. Can J Anaesth 2011 Nov;58(11):1029-1033.

2. Krishnan H, May RE. An unusual cause for respiratory difficulty after thyroidectomy. Br J Clin Pract 1993 Jan-Feb;47(1):47-48.

3. ElBashier EM, Hassan Widtalla AB, ElMakki Ahmed M. Tracheostomy with thyroidectomy: indications, management and outcome: a prospective study. Int J Surg 2008 Apr;6(2):147-150.

4. Randolph GW, Shin JJ, Grillo HC, Mathisen D, Katlic MR, Kamani D, Zurakowski D. The surgical management of goiter: part II. Surgical treatment and results. Laryngoscope 2011 Jan; 121(1):68-76.

5. Agarwal A, Mishra AK, Gupta SK, Arshad F, Agarwal A, Tripathi M, Singh PK. High incidence of tracheomalacia in longstanding goiters: experience from an endemic goiter region. World J Surg 2007 Apr;31(4):832-837.

6. Abdel Rahim AA, Ahmed ME, Hassan MA. Respiratory complications after thyroidectomy and the need for tracheostomy in patients with a large goiter. Br J Surg 1999 Jan;86(1):88-90.

7. McHenry CR, Piotrowski JJ. Thyroidectomy in patients with marked thyroid enlargement: airway management, morbidity, and outcome. Am Surg 1994 Aug;60(8):586-591.

8. Ağaoğlu N. Tracheopexy for tracheomalacia complicating a giant multinodular goiter: a case report. Acta Chir Belg 2007 Jul-Aug;107(4):460-461.

9. Chi SY, Wu SC, Hsieh KC, Sheen-Chen SM, Chou FF. Noninvasive positive pressure ventilation in the management of post-thyroidectomy tracheomalacia. World J Surg 2011 Sep; 35(9):1977-1983. 